\title{
Morphometric analysis in three-dimensional intracoronary ultrasound: An in vitro and in vivo study performed with a novel system for the contour detection of lumen and plaque
}

\author{
Clemens von Birgelen, MD, Carlo Di Mario, MD, PhD, Wenguang Li, MSc, Johan C.H. Schuurbiers, BSc, \\ Cornelis J. Slager, MSc, Pim J. de Feyter, MD, PhD, Jos R.T.C. Roelandt, MD, PhD, and \\ Patrick W. Serruys, MD, PhD Rotterdam, The Netherlands
}

\begin{abstract}
Currently, automated systems for quantitative analysis by intracoronary ultrasound (ICUS) are restricted to the detection of the lumen. The aim of this study was to determine the accuracy and reproducibility of a new semiautomated contour detection method, providing off-line identification of the intimal leading edge and external contour of the vessel in three-dimensional ICUS. The system allows cross-sectional and volumetric quantification of lumen and of plaque. It applies a minimum-cost algorithm and the concept that edge points derived from previously detected longitudinal contours guide and facilitate the contour detection in the crosssectional images. A tubular phantom with segments of varlous luminal dimensions was examined in vitro during five catheter pull-backs $(1 \mathrm{~mm} / \mathrm{sec})$, and subsequently 20 diseased human coronary arteries were studied in vivo with 2.9F $30 \mathrm{MHz}$ mechanical ultrasound catheters (200 images per $20 \mathrm{~mm}$ segment). The ICUS measurements of phantom lumen area and volume revealed a high correlation with the true phantom areas and volumes $(r=0.99)$; relative mean differences were $-0.65 \%$ to $3.86 \%$ for the areas and $0.25 \%$ to $1.72 \%$ for the volumes of the various segments. Intraobserver and interobserver comparisons showed high correlations ( $r=0.95$ to 0.98 for area and $r=0.99$ for volume) and small mean relative differences $(-0.87 \%$ to $1.08 \%)$, with SD of lumen, plaque, and total vessel measurements not exceeding $7.28 \%, 10.81 \%$, and $4.44 \%$ (area) and $2.66 \%, 2.81 \%$, and $0.67 \%$ (volume), respectively. Thus the proposed analysis system provided accurate measurements of phantom dimensions and can be used to perform highly reproducible area and volume measurements in three-dimensional ICUS in vivo. (Am Heart $J$ 1996;132:516-27.)
\end{abstract}

From the Thoraxcenter, Division of Cardiology, University Hospital Rotterdam, Dijkzigt, Erasmus University, The Netherlands.

Received for publication November 27, 1995; accepted January 4, 1996.

Reprint requests: Patrick W. Serruys, $\mathrm{MD}, \mathrm{PhD}$, Thoraxcenter, Interventional Cardiology, P.O. Box 1738, University Hospital Rotterdam, Dijkzigt, 3000 DR Rotterdam, The Netherlands.

Copyright $(\mathbb{0} 1996$ by Mosby-Year Book, Inc.

$0002-8703 / 96 / \$ 5.00+0 \quad 4 / 1 / 73663$
Intracoronary ultrasound (ICUS) provides arterial cross-sectional images and allows diameter and area measurements of coronary lumen and plaque., ${ }^{1,2}$ These measurements have usually been limited to manual contour tracing of cross-sectional images at the site of the reference segment and the target stenosis. In parallel with the progress in quantitative angiography techniques, which started with manual caliper assessment and finally reached computer-assisted methods, ${ }^{3,4}$ fully automated methods of quantitative analysis of ICUS have been developed to reduce the time of analysis and the subjectivity of manual tracing. ${ }^{5}$ These automated systems can be rapidly applied on-line and provide a survey of vascular structure for clinical decision-making. $6,7,8$

Because the automated quantitative analysis of these programs is restricted to the detection of the lumen and because their success rate frequently is limited, ${ }^{9}$ a semiautomated system for off-line ICUS analysis of atherosclerotic coronary segments was developed to detect the intimal leading edge and the external vessel contour on all of the individual crosssectional images, with use of the complete three-dimensional data set obtained during a motorized pull-back of the ICUS transducer. ${ }^{10}$ This method permits volumetric quantification of vessel dimensions by compiling information obtained from the individual cross-sectional images.

The present study was performed to determine the accuracy of this contour detection method in tubular phantoms of known dimensions in vitro and to evaluate intraobserver and interobserver variabilities of area and volume measurements in diseased human coronary arteries in vivo. 
Table I. Characteristics of patients and coronary artery segments

$\begin{array}{lc}\text { Patients }(n) & 20 \\ \text { Age (yr) } & 52 \pm 9.4 \\ \text { Men }(n) & 16(80 \%) \\ \text { Vessels }(n) & \\ \text { LAD } & 15(75 \%) \\ \text { LCX } & 1(5 \%) \\ \text { RCA } & 4(20 \%) \\ \text { Segments }(n) & 11(55 \%) \\ \text { Proximal } & 8(40 \%) \\ \text { Middle } & 1(5 \%) \\ \text { Distal } & \\ \text { Intervention status }(n) & 3(15 \%) \\ \text { Before intervention } & 1(5 \%) \\ \text { Control after HTX } & 2(10 \%) \\ \text { After PTCA } & 3(15 \%) \\ \text { Follow-up PTCA } & 3(15 \%) \\ \text { After DCA } & 5(25 \%) \\ \text { Follow-up DCA } & 3(15 \%) \\ \text { After stenting } & \end{array}$

$D C A$, Directional coronary atherectomy; $H T X$, heart transplantation; $L A D$, left anterior descending coronary artery; $L C X$, left circumflex coronary artery; PTCA, percutaneous transluminal coronary angioplasty; $R C A$, right coronary artery.

\section{METHODS}

Phantom study in vitro. A tubular paraffin phantom was constructed and fixed inside an acrylate tube. The phantom has a circular lumen with a stepwise increase in diameter $(2,3,4$, and $5 \mathrm{~mm}$ ) defining four segments ( $\mathrm{S} 2$, $\mathrm{S} 3, \mathrm{~S} 4$, and S5, respectively) each $5 \mathrm{~mm}$ in length (Fig. 1). A paraffin phantom was used because its properties with regard to the reflection and absorption of ultrasound are similar to those of vessel tissue. An optical calibration was performed with a calibrated stereomicroscope with 40 -fold magnification for the $2 \mathrm{~mm}$ segment and sixteenfold magnification for all of the remaining segments. The mean difference between measurements and the true lumen diameter was $-15 \pm 41 \mu \mathrm{m}$ at $20^{\circ} \mathrm{C}$, the temperature at which the experiments were performed. Temperature dependency of the paraffin phantom was assessed by comparing measurements obtained at $2^{\circ}$ and $37^{\circ} \mathrm{C}$ (range $35^{\circ} \mathrm{C}$ ). This temperature increment resulted in an increase in luminal dimensions of $2 \%$.

Five motorized uniform pull-backs $(1 \mathrm{~mm} / \mathrm{sec})$ of the ultrasound imaging transducer through the paraffin phantom were performed in water $\left(20^{\circ} \mathrm{C}\right)$ and recorded on videotape. A mechanical rotating ICUS catheter (Microview, Cardiovascular Imaging Systems, Inc., Sunnyvale, Calif.) with a distal external diameter of $2.9 \mathrm{~F}$ was used for both in vitro and in vivo studies. This ICUS imaging catheter is equipped with a distal transparent sleeve that covers the rotating imaging core. With a motorized pull-back system the imaging core is withdrawn inside this sleeve. The design of the catheter and pull-back system minimizes the risk of catheter rotation and facilitates several pull-backs of the echo transducer without increasing the risk of vessel damage, because the echo-transparent distal sleeve

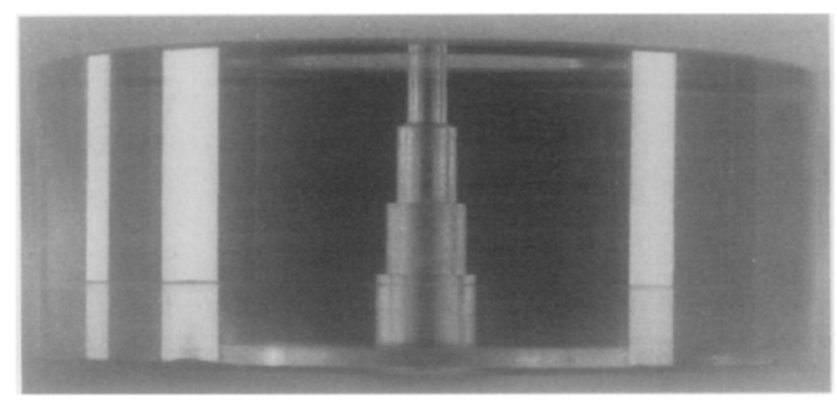

Fig. 1. Model of paraffin phantom used in in vitro study. Phantom has circular lumen and consists of four segments with stepwise increase in diameter $(2,3,4$, and $5 \mathrm{~mm})$.

prevents the ICUS transducer from coming into direct contact with the vessel wall.

Study in vivo. Intraobserver and interobserver variabilities of the quantification method were studied in 20 ICUS examinations of diseased, nonwedged human coronary segments in vivo. Segments with short calcifications or single major side branches were included in the study; ICUS studies with excessive systolic-diastolic movement were not considered for analysis. The maximum and average cross-sectional area obstructions were $65.6 \% \pm$ $8.8 \%$ (range $83 \%$ to $49 \%$ ) and $49.2 \% \pm 6.6 \%$ (range $83 \%$ to $17 \%$, respectively. The composition of the study population reflects the current clinical application of ICUS imaging in our center. Characteristics of the patients and analyzed coronary artery segments are shown in Table I.

ICUS imaging was performed during motorized pullbacks $(1 \mathrm{~mm} / \mathrm{sec}$ ) of a 2.9 F ICUS catheter. Because the imaging core is straightened during the first seconds of withdrawal, care was taken to start the pull-back $1 \mathrm{~cm}$ distal to the segment analyzed. The ICUS examinations were recorded on videotape, and analysis was performed off-line by a new quantitative ultrasound analysis system set at a digitization frame rate of 10 images/sec. Thus $20 \mathrm{~mm}$-long coronary artery segments were reconstructed and measured using the system's maximum memory capacity, which is currently 200 images.

The quantitative ICUS analysis system. The analysis program uses the Microsoft (Redmond, Wash.) Windows operating system on a Pentium (Intel)-based $60 \mathrm{MHz}$ personal computer with $16 \mathrm{Mb}$ internal random-access memory. A frame-grabber is installed (DT-3852, Data Translation, Inc., Malboro, Mass.; resolution $800 \times 600 \times 8$ bits), digitizing a user-defined region of interest from the video images. A maximum of 200 ICUS images can be digitized at a user-defined digitization frame rate (maximum 20 images/sec). The reconstructed segment length is thus defined by the speed of the motorized pull-back during the basic image acquisition and by the digitization frame rate. In the present study a pull-back speed of $1.0 \mathrm{~mm} / \mathrm{sec}$ and a digitization frame rate of 8 images/sec (in vitro) and 10 images/sec (in vivo) were used, resulting in reconstructed segment lengths of 25 and $20 \mathrm{~mm}$, respectively. The pixel size, which depends on the magnification applied by the basic ICUS imaging system, ranged from 26 to $36 \mu \mathrm{m}$. 


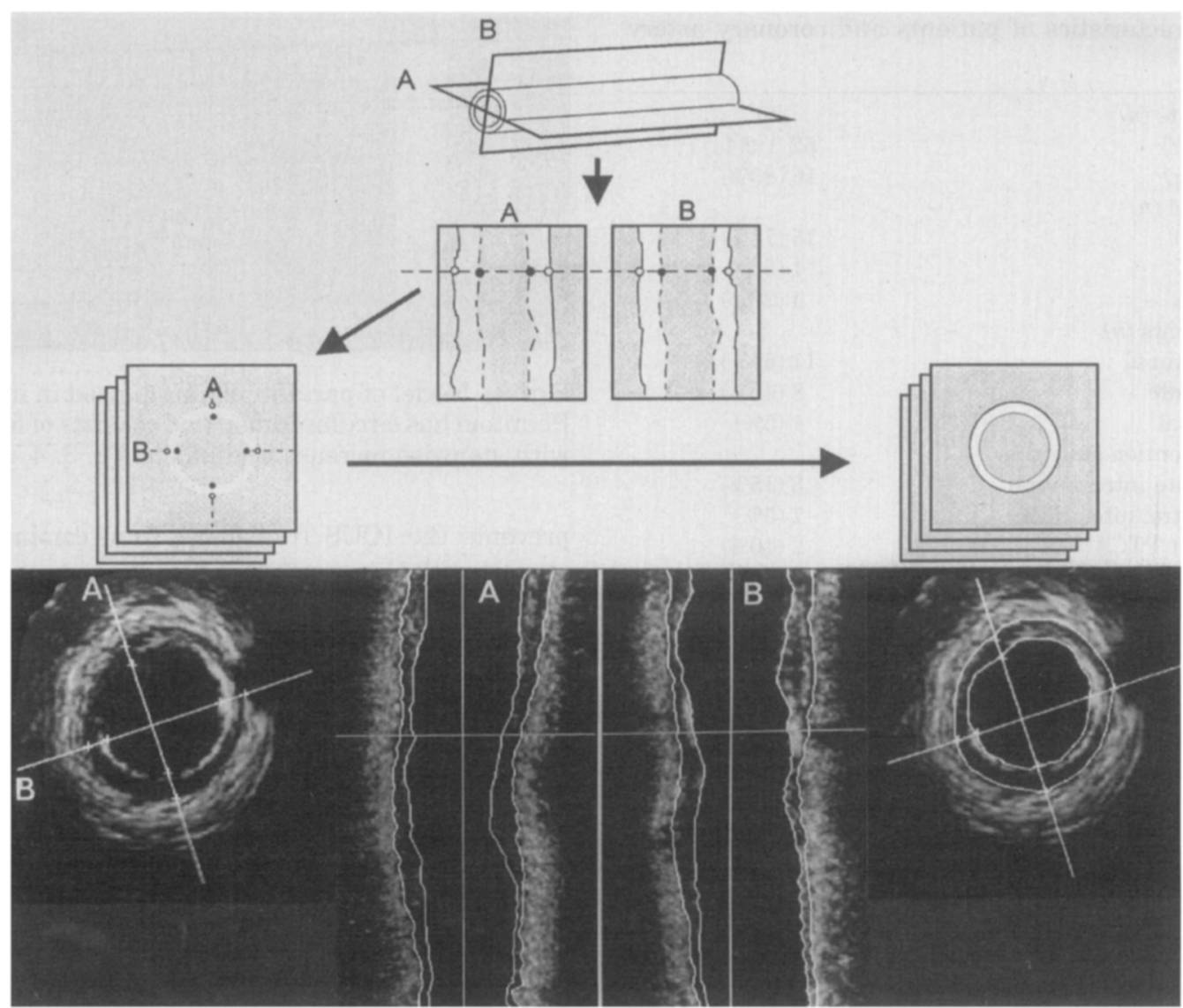

Fig. 2. Morphometric analysis by contour detection in three-dimensional ICUS. With this method, edge points derived from longitudinal contours previously detected on two longitudinally reconstructed images guide and facilitate final contour detection on transverse ICUS images. ICUS images, obtained during motorized pull-back, are stored in voxel space. Two perpendicular cut planes $(A$ and $B)$ that are placed interactively are used to reconstruct two longitudinal sections from ICUS image data located at intersections. Automated contour detection is performed in these longitudinal sections on basis of application of minimum-cost algorithm. User then is free to set some markers on longitudinal images to force contours to pass through these sites, and optimal path is redefined by dynamic programming techniques. Longitudinal contours are updated during entire interactive procedure and are represented as individual edge points in transverse images; these points guide contour detection on basis of application of minimum-cost algorithm. Position of an individual transverse plane in longitudinal sections is indicated by horizontal cursor line, which can be used to scroll through whole series of transverse images. Finally, detected contours are checked in all of transverse images, and manual correction of contours can be performed.

Minimum-cost algorithm. The contour detection of the intimal leading edge and the external boundary of the total vessel applies a minimum-cost algorithm, previously applied $^{11}$ and described ${ }^{12}$ in cross-sectional ICUS images. By this approach the digitized ICUS images are resampled according to a radial image reconstruction (64 radii in the cross-sectional images; 200 rows in the longitudinal sections). A cost matrix that represents the edge strength is calculated from the image data. For the detection of the boundary between lumen and plaque, the cost value is defined by the spatial first derivative. To detect the external boundary of the total vessel a pattern-matching process by cross correlation is adopted for the cost calculations. Through the cost matrixes a path with the smallest accu- mulated value is determined by dynamic programming techniques. $^{11}$

Automated contour detection. For contour detection of the intimal leading edge and the external boundary of the total vessel, three steps must be performed because, in this system, edge points, derived from previously detected longitudinal contours, guide and facilitate the final contour detection in the cross-sectional ICUS images.

First, a sequence of digitized ICUS images obtained from the motorized pull-back of the ultrasound transducer is stored in a voxel space. ${ }^{13}$ Ringdown artifacts around the ICUS catheter, potentially interfering with the contour detection step, can be removed from all of the ICUS images with an automated function. Two perpendicular cut planes 


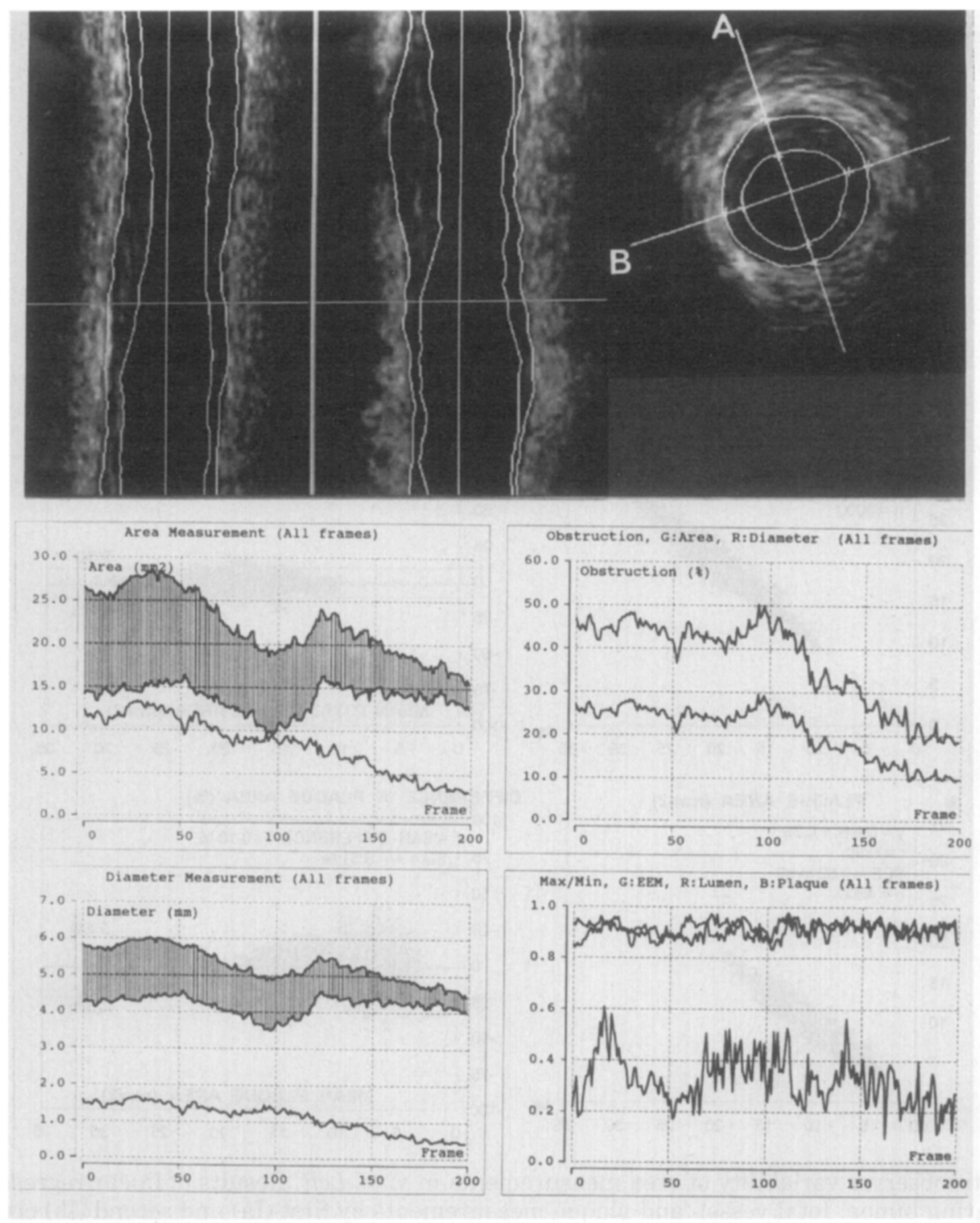

Fig. 3. Standard display of results. This clinical example shows results of quantitative analysis performed at 6-month follow-up after directional coronary atherectomy performed in proximal left anterior descending coronary artery. The intimal leading edge and external vessel contours are shown in two longitudinally reconstructed sections ( $A$ and $B$, left and mid top), which stand perpendicular to each other as demonstrated in the transverse image (right top). Left middle and left bottom, Area and mean diameter measurements of lumen, total vessel, and plaque. Gray areas represent coronary plaque, and site of maximal plaque burden can thus be easily identified. Upper and lower boundaries of gray zone correspond to dimensions of coronary lumen and total vessel. Absolute value of plaque dimension is given as single function in display of area and in diameter measurements. Right middle, Functions of relative diameter obstruction and area obstruction. Right bottom, Symmetry ratios of both contours and plaque eccentricity ratio.

running along the long axis of the artery are used to reconstruct two longitudinal sections (Fig. 2). This longitudinal reconstruction uses the ICUS image data, located at the intersection of the cut planes with the voxel space. The position and the rotation angle of the two cut planes can be changed interactively by the user to obtain an optimal representation of the reconstructed coronary segment in the longitudinal sections.

Second, the longitudinal contours are detected in these two longitudinal images. A first boundary detection is performed automatically on the basis of the application of the minimum-cost algorithm. Then the user is free to set some markers in the longitudinal images and to force the contours to pass through these sites. This step is achieved by setting the cost matrix of the manually defined sites at a very low value. By applying dynamic programming techniques, the optimal path is then redefined for the modified cost matrix. During the entire user-interactive procedure 

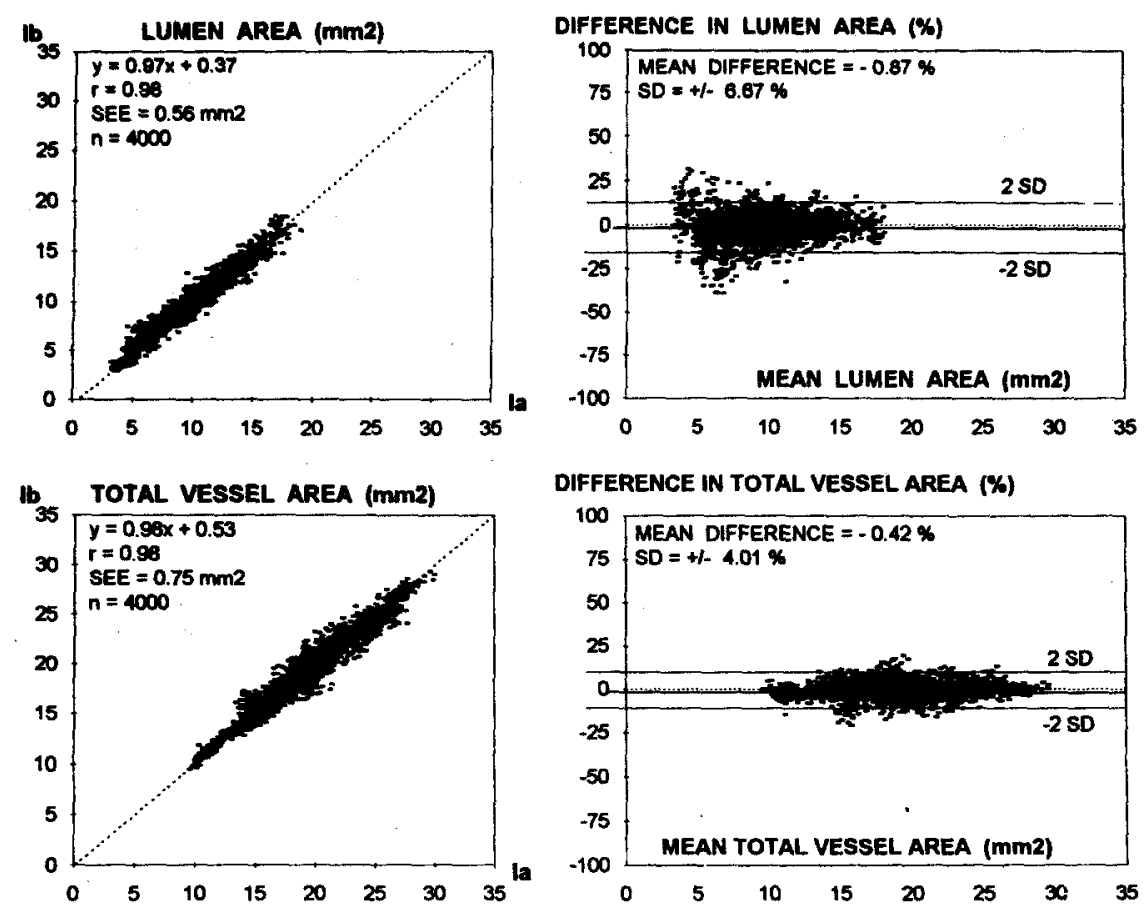

DIFFERENCE IN TOTAL VESSEL AREA (\%)
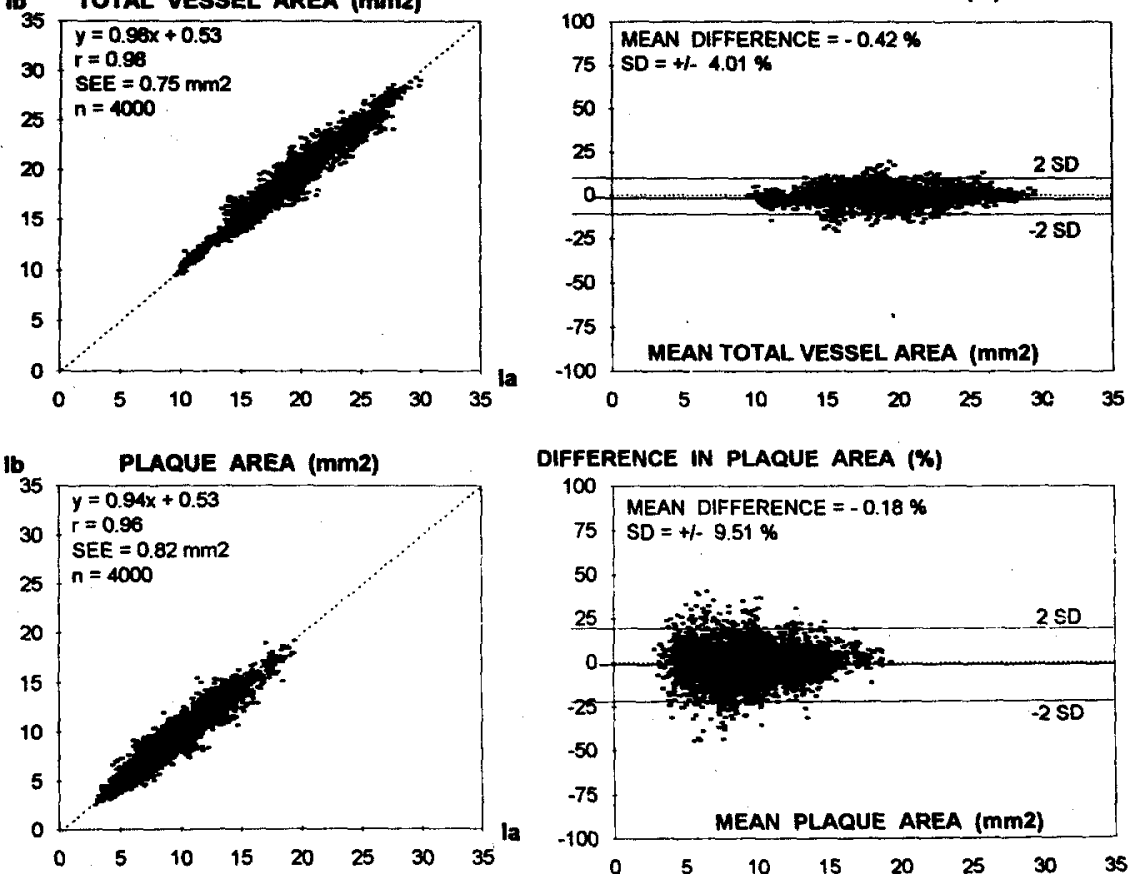

Fig. 4. Intraobserver variability of area measurements in vivo. Left, Results of linear regression analyses, comparing lumen, total vessel, and plaque measurements by first (Ia) and second (lb) observations. Right, Relative intraobserver differences plotted against mean of two measurements. Continuous lines, Relative mean signed difference and $2 \mathrm{SD}$; dotted line, line of identity.

the longitudinal contours are visible and updated in the longitudinal sections. In the transverse images the longitudinal contours intersecting this plane are represented as points.

These individual edge points guide the third step, which is the final contour detection in the transverse images, by defining the center and range of the boundary searching process on the basis of the application of the minimum-cost algorithm. The position of an individual transverse plane in the longitudinal sections is indicated by a horizontal cursor line, which can be used to scroll through the whole series of transverse images. The detected contours are checked by the analyst in all of the transverse images, and manual correction of the contours can be performed.

Calculation and display of results. The quantitative results, including diameter and area measurements of lumen, total vessel, and plaque; percentage diameter ob- struction; and percentage area obstruction are displayed (Fig. 3). Plaque area is calculated by subtracting the lumen area from the total vessel area, thus representing the plaque-media complex. Volumes of lumen, total vessel, or plaque áre calculated as

$$
\mathrm{V}=\sum_{\mathbf{i}=1}^{n} \mathrm{~A}_{\mathbf{i}} \cdot \mathbf{H}
$$

where $\mathrm{V}=$ volume; $\mathrm{A}=$ area of lumen, total vessel, or plaque in a given digitized cross-sectional ultrasound image; $\mathbf{H}=$ the thickness of the coronary artery slice, which is represented by this digital cross-sectional ICUS image; and $n=$ the number of digitized cross-sectional images encompassing the volume to be measured.

Mean values, $S D_{s}$, and minimum and maximum values of area and volume measurements are presented. The data of the current transverse image are constantly displayed 

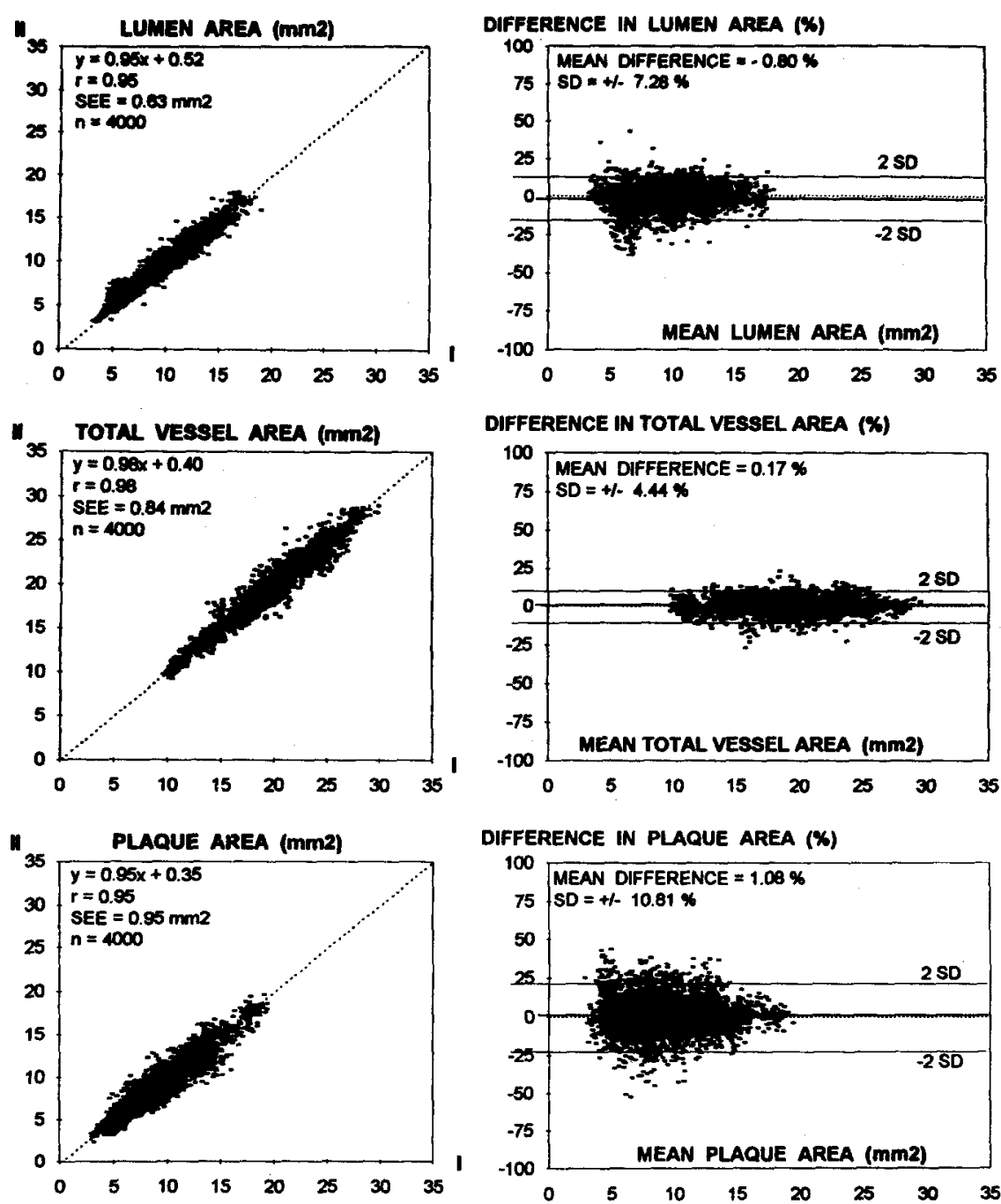

Fig. 5. Interobserver variability of area measurements in vivo. Left, results of linear regression analyses, comparing lumen, total vessel, and plaque measurements by first (Ia) and second (II) observers. Right, Relative interobserver differences plotted against mean of two measurements. Continuous lines, Relative mean signed difference and $2 \mathrm{SD}$; dotted line, line of identity.

and serially updated if manual corrections of the contours are performed. The analyzed artery segment can be displayed in a three-dimensional, cylindrical format; however, this format is not required for the quantification process that uses the three-dimensional data set (see Discussion section).

Data analysis. At the transition between two phantom segments the circular contours of the two adjacent segments are simultaneously visualized by ICUS because of the limited out-of-plane resolution of the current ultrasound transducers. ${ }^{14}$ In this study, when the ultrasound transducer was withdrawn from the segment with the smaller dimension to the segment with the larger dimension, the transition to the larger segment was defined as the first image in which the contour of the larger segment expressed a higher intensity than the contour of the smaller segment. This definition was used to calculate the volumes of the phantom segments. For validation of the area measurements, images from the mid-portion of each segment were used.

ICUS imaging systems are calibrated for application in blood, but the in vitro experiments of the present study were performed in water. Accordingly, a correction factor (0.953) determined on the basis of the differing velocities of ultrasound in blood $(1570 \mathrm{~m} / \mathrm{sec})$ and water $(1497$ $\mathrm{m} / \mathrm{sec})^{15}$ was applied.

The same digitized in vivo ICUS images were analyzed off-line by two independent observers, who had had common training in the use of the semiautomated contour detection system. After 2 to 3 weeks the analysis was repeated by the blind, first observer. The measurements by two independent observers (Ia and II) and the repeated 
Table II. Coronary artery lumen, total vessel, and plaque volume in vivo: Results of the intraobserver and interobserver studies

\begin{tabular}{|c|c|c|c|c|c|c|c|c|c|}
\hline \multirow[b]{2}{*}{ Patient } & \multicolumn{3}{|c|}{ Lumen $\left(\mathrm{mm}^{3}\right)$} & \multicolumn{3}{|c|}{ Total vessel $\left(\mathrm{mm}^{3}\right)$} & \multicolumn{3}{|c|}{ Plaque $\left(\mathrm{mm}^{3}\right)$} \\
\hline & $I a$ & $I b$ & $I I$ & Ia & $I b$ & $I I$ & $I a$ & $I b$ & $I I$ \\
\hline 1 & 158.3 & 159.0 & 157.8 & 338.9 & 336.0 & 332.6 & 180.6 & 177.0 & 174.8 \\
\hline 2 & 185.5 & 193.4 & 197.6 & 427.9 & 430.7 & 430.6 & 242.4 & 237.3 & 233.0 \\
\hline 3 & 159.5 & 163.4 & 160.1 & 335.3 & 337.4 & 337.1 & 175.8 & 174.0 & 177.0 \\
\hline 4 & 208.4 & 203.0 & 207.7 & 439.9 & 439.0 & 441.3 & 231.5 & 236.0 & 233.6 \\
\hline 5 & 207.8 & 214.1 & 200.3 & 400.5 & 407.9 & 400.6 & 192.7 & 193.8 & 200.3 \\
\hline 6 & 202.0 & 199.2 & 206.0 & 446.2 & 447.2 & 445.4 & 244.2 & 248.0 & 239.4 \\
\hline 7 & 204.8 & 211.3 & 204.3 & 404.9 & 411.8 & 406.1 & 200.1 & 200.5 & 201.8 \\
\hline 8 & 216.6 & 214.2 & 204.9 & 375.8 & 376.0 & 372.1 & 159.2 & 161.8 & 167.2 \\
\hline 9 & 231.1 & 233.5 & 231.7 & 431.6 & 431.8 & 427.7 & 200.5 & 198.2 & 196.0 \\
\hline 10 & 262.7 & 261.0 & 261.0 & 456.3 & 454.8 & 452.9 & 193.6 & 193.7 & 191.9 \\
\hline 11 & 145.2 & 153.5 & 154.1 & 292.8 & 294.6 & 290.3 & 147.6 & 141.2 & 136.2 \\
\hline 12 & 168.7 & 168.6 & 172.4 & 341.0 & 342.2 & 343.2 & 172.3 & 173.6 & 170.8 \\
\hline 13 & 129.0 & 131.8 & 132.9 & 271.1 & 272.6 & 270.4 & 142.1 & 140.8 & 137.5 \\
\hline 14 & 130.8 & 129.3 & 130.3 & 360.5 & 362.3 & 360.5 & 229.7 & 233.0 & 230.2 \\
\hline 15 & 107.6 & 105.8 & 109.6 & 215.4 & 216.2 & 214.8 & 107.8 & 110.4 & 105.2 \\
\hline 16 & 211.2 & 213.4 & 214.4 & 444.0 & 447.2 & 443.8 & 232.8 & 233.8 & 229.4 \\
\hline 17 & 190.2 & 191.8 & 190.4 & 419.2 & 421.8 & 422.8 & 229.0 & 230.0 & 232.8 \\
\hline 18 & 204.4 & 205.2 & 205.0 & 363.8 & 366.6 & 362.2 & 159.4 & 161.4 & 157.2 \\
\hline 19 & 193.0 & 193.4 & 193.6 & 293.2 & 292.8 & 292.8 & 100.2 & 99.4 & 99.2 \\
\hline 20 & 275.2 & 275.2 & 276.2 & 443.6 & 445.6 & 442.6 & 168.4 & 170.4 & 166.4 \\
\hline Mean & 189.6 & 191.0 & 190.5 & 375.1 & 376.7 & 374.5 & 185.5 & 185.7 & 184.0 \\
\hline $\mathrm{SD}$ & 42.9 & 42.6 & 41.7 & 68.4 & 68.8 & 68.7 & 42.3 & 42.9 & 42.8 \\
\hline
\end{tabular}

$I$, First observer ( $a$, first observation; $b$, second observation); $I I$, second observer.

measurements by the same observer (Ia and Ib) were studied to obtain information on the intraobserver and interobserver variabilities of the new analysis method.

Statistics. Results are given as mean $\pm \mathrm{SD}$. The mean difference and SD of the differences were calculated for each phantom segment (ICUS measurements minus true phantom dimensions) and for repeated measurements by the same observer and for measurements by two observers. ${ }^{16}$ Analysis of variance and linear regression analysis were performed to compare the results of the intraobserver study and the results of the interobserver study.

\section{RESULTS}

Phantom study in vitro. The lumen area measurements were compared with the true values by linear regression analysis. The measurements $(n=600)$ and true phantom values showed a high correlation $\left(r=0.99 ; \mathrm{y}=0.99 \mathrm{x}+0.11 ; \mathrm{SEE}=0.12 \mathrm{~mm}^{2}\right)$. The mean difference between measurements and true phantom areas ranged from $-0.65 \%$ to $1.24 \%$ with the exception of the smallest segment, in which a small overestimation (3.86\%) was found. Correlation between the volume measurements $(n=20)$ and the true phantom volumes was high $(r=0.99$; $\mathrm{y}=1.02 \mathrm{x}-0.42 ; \mathrm{SEE}=1.17 \mathrm{~mm}^{3}$ ). The measured volumes showed a slight overestimation; the mean difference ranged from $0.25 \%$ to $1.72 \%$. A decrease in phantom dimensions was associated with a slight increase in the relative SD of the difference of area and volume measurements $(0.56 \%, 0.95 \%, 0.95 \%$, and $2.55 \%$ for area and $1.40 \%, 2.69 \%, 2.78 \%$, and $3.75 \%$ for volume measurements in the segments with lumen diameters of $5,4,3$, and $2 \mathrm{~mm}$, respectively).

Area measurement in vivo. The time required for the complete analysis was $1.15 \pm 0.31$ hours. The correlation of repeated area measurements of coronary lumen, total vessel, and plaque was high, with correlation coefficients of $0.98,0.98$, and 0.96 respectively (Fig. 4, left). The mean relative differences between repeated area measurements by the same observer were $<1 \%$ for lumen, total vessel, and plaque areas (Fig. 4, right). The SD of the differences was higher for the plaque area than for lumen and total vessel areas $(9.51 \%$ vs $6.67 \%$ and $4.01 \%$, respectively). The interobserver correlation coefficients were high ( $r=0.95,0.98$, and 0.95 for lumen, total vessel, and plaque, respectively) (Fig. 5, left), but for lumen and plaque area measurements they were slightly lower than the corresponding intraobserver correlation coefficients. The interobserver SEEs of lumen, total vessel, and plaque areas $(0.63,0.84$, and $0.95 \mathrm{~mm}^{2}$, respectively) also were higher than the 

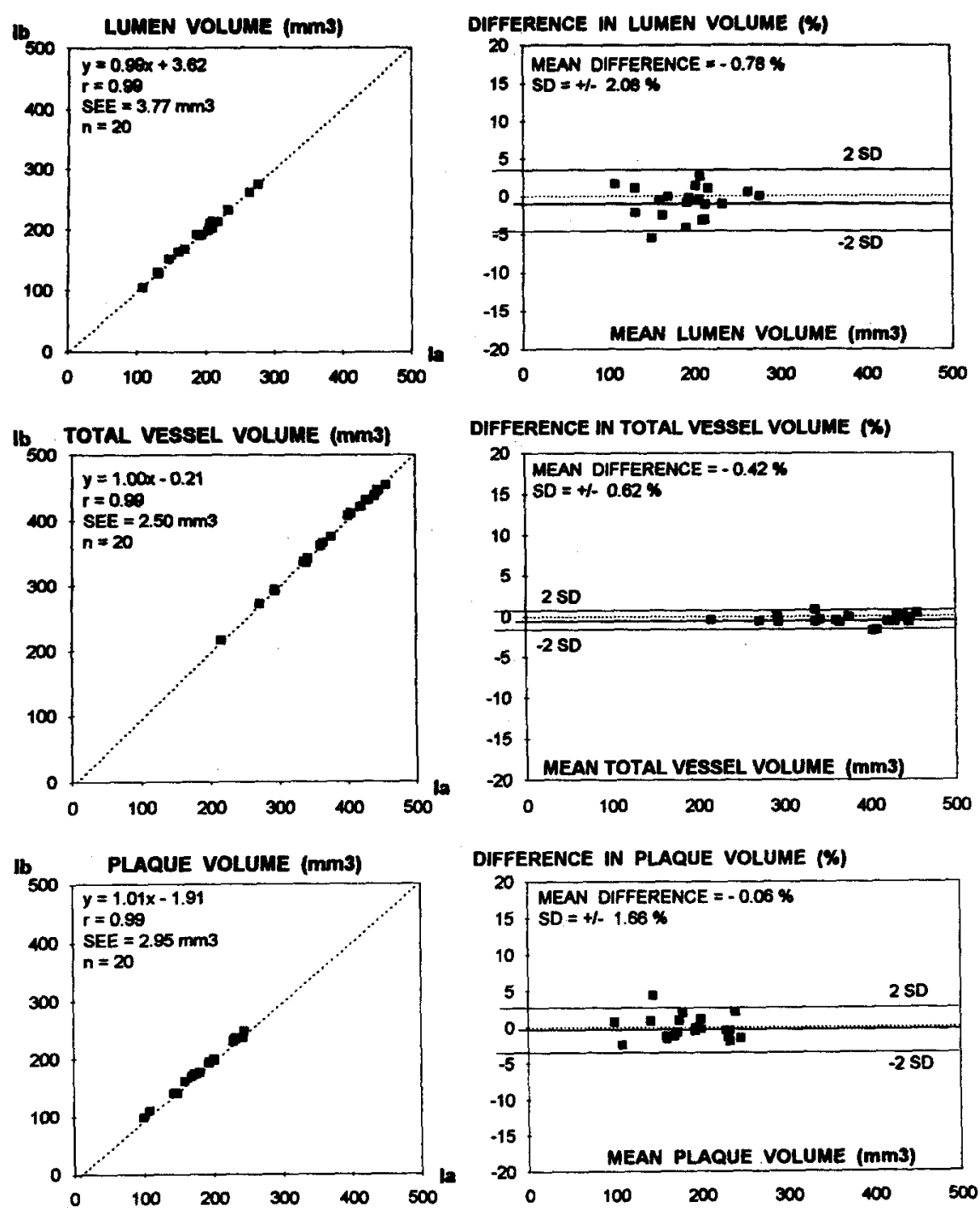

Fig. 6. Intraobserver variability of volume measurements in vivo. Left, Results of linear regression analyses, comparing lumen, total vessel, and plaque measurements of first (Ia) and second (Ib) observations. Right, Relative intraobserver differences plotted against mean of two measurements. Continuous lines, Relative mean signed difference and 2 SD; dotted line, line of identity.

corresponding intraobserver SEEs. The SD of the relative differences were slightly higher for interobserver differences (Fig. 5, right) than for intraobserver differences. The data points for smaller dimensions of lumen and plaque areas showed a larger relative dispersion.

Volume measurement in vivo. The results of the lumen, total vessel, and plaque volume measurements are shown in Table II. A high correlation between the intraobserver measurements was found, with a correlation coefficient of 0.99 for coronary lumen, total vessel, and plaque (Fig. 6, left). The correlation coefficients also were high for the comparison of the measurements by two independent observers ( $r=0.99$ for lumen, total vessel, and plaque volumes)
(Fig. 7, left). The SEE and the SD of the differences were higher for the interobserver than for the intraobserver comparison (Figs. 6, right, and 7, right).

\section{DISCUSSION}

The application of high-frequency ICUS permits assessment of catheter-based interventions ${ }^{17-20}$ and visualization (Fig. 8) and quantification of coronary artery atherosclerosis. ${ }^{21-24}$ Serial ICUS studies also permit assessment of the progression or regression of atherosclerosis and of the mechanisms of coronary restenosis. $^{25}$

The main limitation in the comparison of serial ultrasound studies is the virtual impossibility of examining exactly the same ultrasonic cross section in 

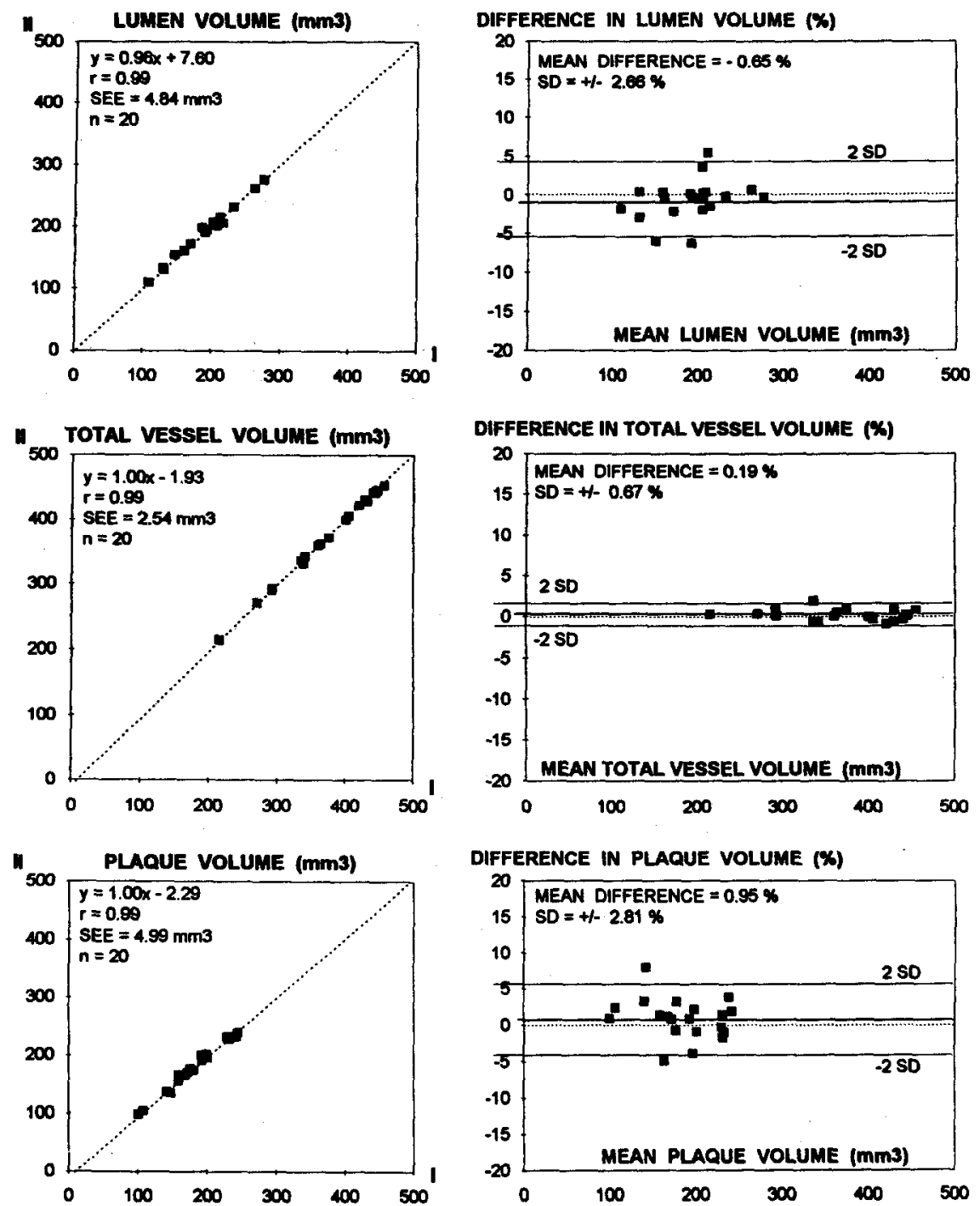

Fig. 7. Interobserver variability of volume measurements in vivo. Left, Results of linear regression analyses, comparing lumen, total vessel, and plaque measurements of first (Ia) and second (II) observers. Right, Relative interobserver differences plotted against mean of two measurements. Continuous lines, Relative mean signed difference and $2 \mathrm{SD}$; dotted line, line of identity.

each study. In previous studies, anatomic landmarks such as side branches or deep calcifications were used to define corresponding images in serial studies. The application of the proposed analysis system. offers a more reliable solution to this problem, because a long arterial segment can be examined. Minimal differences in the start and end points of repeated studies are unlikely to impair the accuracy of the changes in lumen and plaque volume measurements, assessed for an entire arterial segment. Because the aim of this study was to determine the accuracy and reproducibility of the analysis method, the same set of digitized images from a single pullback per patient was used for repeated analysis.
The results of the in vitro study suggest that the automated analysis system operates accurately, and the in vivo study demonstrates high reproducibility. The SDs of the intraobserver and interobserver differences in detecting the total vessel contours were particularly low, reflecting the regular shape of the external boundary of the total vessel. The lumen contours, however, demonstrated slightly higher variability, which can be explained by irregularities in the shape of the lumen area, especially after coronary interventions. The larger variability of plaque area measurements, derived from measurements of lumen and total vessel contours, reflect the combined variability of these two contours. The volumetric 
measurements in vivo showed a lower SD of the differences than did the area measurements, reflecting an averaging of the differences of the area measurements.

The ability to display the cross-sectional plus two longitudinal views simultaneously facilitates the detection of the boundary of the vessel. The reason for first performing contour detection on the longitudinal images is that the edge information obtained from this step is subsequently used to guide the final contour detection on the transverse images.

Previous studies. A different approach to contour detection, providing automated tracking of the coronary wall and lumen in ICUS image sequences, has been developed by Sonka et al. ${ }^{26}$ The border tracking is performed only in the cross-sectional images, without the assistance of an additional contour detection step in longitudinally reconstructed images. The feasibility of this system in analyzing in vivo examinations remains to be confirmed because the movement of the ICUS catheter or the poor depiction of the plaque in single ICUS images may impair the application of this algorithm, but the first results in vascular specimen in vitro are promising.

Data on the variability and reproducibility of ICUS measurements are limited to the assessment of selected individual cross sections in in vitro and in vivo studies and, in general, address different questions. A recent systematic study ${ }^{5}$ on the variability of measurements in 120 cross-sectional ICUS images in vivo demonstrated relatively high variability of the manual contour tracing. ${ }^{27}$ In agreement with the present study, the SD of the differences was higher for plaque than for vessel lumen or total area measurement. However, the variability of repeated manual contour tracing 5 was indeed higher than the variability of the proposed analysis system in the present study. This difference may be explained in part by the use of larger ultrasound catheters in the previous study ${ }^{27}$, which show strut artifacts, obscuring a portion of the vessel wall, and the use of single digitized frames without the option of scrolling the videotape back or forth.

Information about the reproducibility of volumetric measurements is limited, and it is difficult to compare results provided by different quantitative analysis systems. Matar et al. ${ }^{28}$ recently reported an intraobserver study that yielded a correlation coefficient of 0.98 for automated threshold-based measurement of lumen volume in vivo, a result confirmed by the high reproducibility of volumetric measurement observed in the present study.

Approaches toward volumetric quantification. Threedimensional ICUS was first used clinically to assess

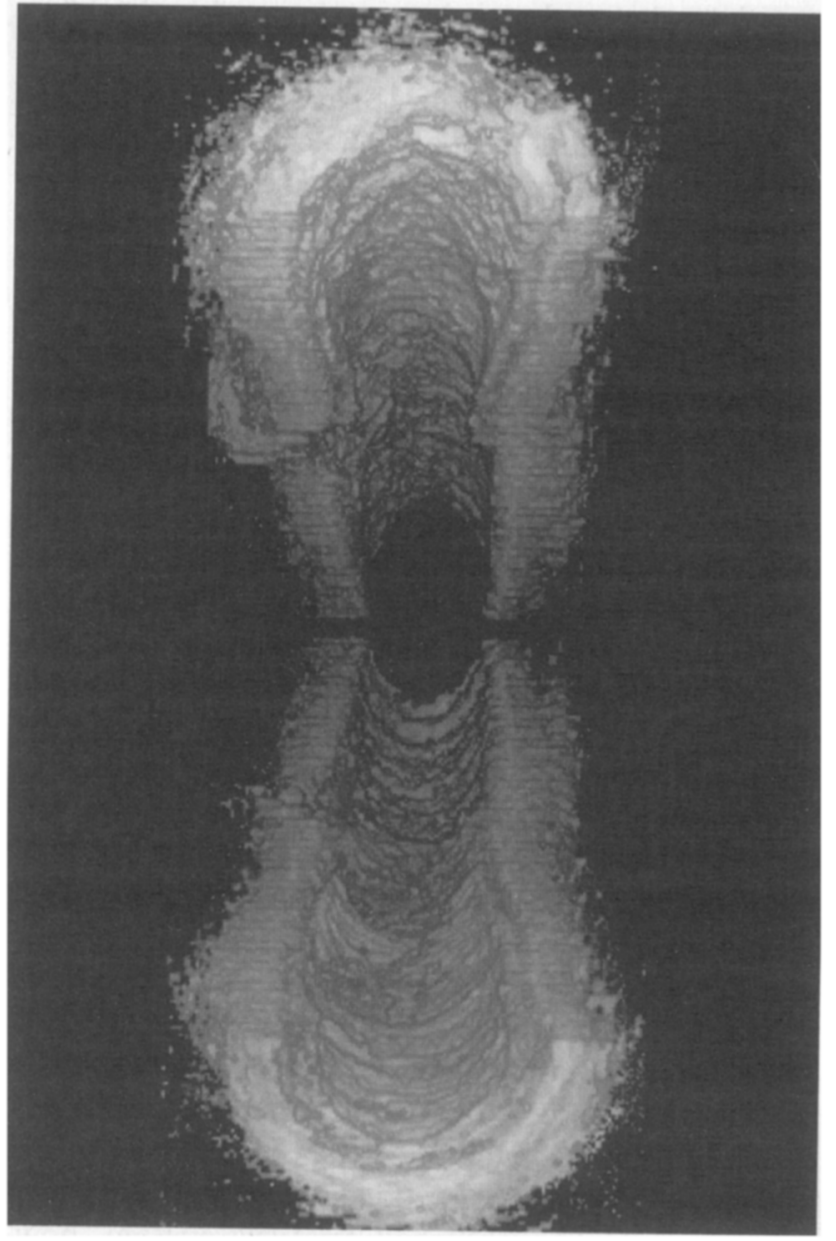

Fig. 8. Three-dimensional display of coronary segment at follow-up after directional coronary atherectomy. This display uses cylindrical format; it is not required for quantification from three-dimensional data set. Nevertheless, it may provide additional insight into plaque geometric features.

visually the spatial configuration of plaques. $^{29}$ The morphologic features of dissections ${ }^{30,31}$ and stents $^{32,33}$ were examined, and their length or diameter was measured. However, the three-dimensional data set can also be processed by various technical approaches to quantify lumen or plaque volumes. These systems differ in terms of image segmentation, which is the algorithm for identification of the structures of interest in the digitized ICUS images. Distinction between vessel lumen and wall can be achieved by various (semi)automated systems on-line with binary threshold-based algorithms, ${ }^{28,}{ }^{29}$ voxel-based segmentation algorithms, ${ }^{34}$ or algorithms for statistical pattern recognition..$^{6-8}$ The applicability of these approaches depends considerably on image quality and is restricted to the automated detection of the lumen. The proposed quantitative analysis system, however, combines automatic con- 
tour detection and user interaction and is therefore able partly to compensate for limitations in ICUS image quality. The system is capable of identifying the intimal leading edge and the external contour of the vessel, thus permitting automated quantification of plaque volume.

The possibility of examining the vascular segment at the same time in a transverse and two longitudinal views and the concept of applying an automated contour detection in the cross-sectional ICUS images, guided by edge points derived from previously detected longitudinal contours, are the key factors explaining the high reproducibility of this ICUS analysis method in vivo.

Contradictory results have been observed in previous progression-regression trials using quantitative coronary angiography. ICUS directly visualizes the vessel wall and therefore may better reflect the changes of the plaque, but in progression-regression studies small changes can be expected despite the long study period, and a high reproducibility of measurements is important. On the basis of the results of the present study, which demonstrates high reproducibility of the volumetric ICUS measurement, volumetric ICUS measures may be considered ${ }^{35}$

Limitations. Behind deposits of calcium the external vessel boundary cannot be traced. In addition, depending on the type of stent, the metallic struts may occasionally render the external contour detection difficult. The proposed analysis system, however, allows reliable interpolation between preceding and following images on the longitudinal sections. The edge information finally permits reproducible interpolated contour tracing on the cross-sectional ICUS images. Artifacts in the longitudinally reconstructed views, caused by the movement of the ultrasound catheter during the cardiac cycle and systolic-diastolic changes in vessel dimensions, ${ }^{6,9}$ may in the future be avoided by electrocardiographically (ECG) gated ${ }^{36,37}$ ICUS image acquisition or even the combination of ECG-gated ICUS and angiographic data ${ }^{38,39}$ Application of these combined approaches is still restricted, but the analysis system used in the present study is now used clinically for on-line and off-line analyses, and evaluation of taperecorded ultrasound studies from multicenter trials has recently been started.

Conclusion. The proposed analysis system provides accurate measurements of phantom dimensions and can be used to perform highly reproducible area and volume measurements in three-dimensional ICUS in vivo.

We thank Ellie van de Leur, Bart van der Zalm, and Jurgen Ligthart for technical support.

\section{REFERENCES}

1. Yock $P G$, Linker DT. Intravascular ultrasound: looking below the surface of vascular disease. Circulation 1990;81:1715-8.

2. Nissen SE, Gurley JC, Grines CL, Booth DC, McClure R, Berk M, et al. Intravascular ultrasound assessment of lumen size and wall morphology in normal subjects and patients with coronary artery disease. Circulation 1991;84:1087-99.

3. Keane D, Serruys PW. Quantitative coronary angiography: an integral component of interventional cardiology. In: Topol EJ, Serruys PW, eds. Current review of interventional cardiology. 2nd ed. Philadelphia: Current Medicine, 1995:205-33.

4. von Birgelen C, Umans V, Di Mario C, Keane D, Gil R, Prati F, et al. Mechanism of high-speed rotational atherectomy and adjunctive balloon angioplasty revisited by quantitative coronary angiography: edge detection versus videodensitometry. Am Heart J 1995;130:405-12.

5. Hausmann D, Lundkvist AJS, Friedrich GJ, Mullen WL, Fitzgerald PJ, Yock PG. Intracoronary ultrasound imaging: intraobserver and interobserver variability of morphometric measurements. Am Heart J 1994; 128:674-80.

6. Di Mario C, von Birgelen C, Prati F, Soni B, Li W, Bruining N, et al. Three-dimensional reconstruction of two-dimensional intracoronary ultrasound: clinical or research tool? Br Heart J 1995;73(suppl 2):2632.

7. von Birgelen C, Gil R, Ruygrok P, Prati F, Di Mario C, van der Giessen WJ, et al. Optimized expansion of the Wallstent compared to the Palmaz-Schatz stent: on-line observations with intracoronary ultrasound after angiographic guidance. Am Heart J 1996;131:1067-75.

8. von Birgelen C, Kutryk MJB, Gil R, Ozaki Y, Di Mario C, Roelandt JRTC, et al. Quantification of the minimal luminal cross-sectional area after coronary stenting by two-dimensional and three-dimensional intravascular ultrasound versus edge detection and videodensitometry. Am J Cardiol [In press].

9. Roelandt JRTC, Di Mario C, Pandian NG, Wenguang Li, Keane D, Slager CJ, et al. Three-dimensional reconstruction of intracoronary ultrasound images: rationale, approaches, problems, and directions. Circulation 1994;90:1044-55.

10. Li W, von Birgelen C, Di Mario C, Boersma E, Gussenhoven EJ, van der Putten N, et al. Semi-automatic contour detection for volumetric quantification of intracoronary ultrasound. In: Computers in cardiology 1994. Los Alamitos, Calif.: IEEE Computer Society Press, 1994:27780 .

11. Di Mario C, The SHK, Madretsma S, van Suylen RJ, Wilson RA, Bom $\mathrm{N}$, et al. Detection and characterization of vascular lesions by intravascular ultrasound: an in vitro study correlated with histology. J Am Soc Echocardiogr 1992;5:135-46.

12. Li W, Bosch JG, Zhong Y, van Urk H, Gussenhoven EJ, Mastik F, et al. Image segmentation and $3 \mathrm{D}$ reconstruction of intravascular ultrasound images. In: Wei Y, Gu B, eds. Acoustical imaging. Vol. 20. New York: Plenum Press, 1993:489-96.

13. Kitney RI, Moura L, Straughan K. 3-D visualization of arterial structures using ultrasound and voxel modelling. Int J Cardiac Imaging 1989;4:135-43.

14. Benkeser PJ, Churchwell AL, Lee C, Abouelnasr DM. Resolution limitations in intravascular ultrasound imaging. J Am Soc Echocardiogr 1993;6:158-65.

15. Hertz H. Principles of ultrasound in medical diagnosis. In: Bom N, ed. Echocardiography with Doppler applications and real time imaging. The Hague: Martinus Nijhoff Medical Division, 1977:7-14.

16. Bland JM, Altman DG. Statistical methods for assessing agreement between two methods of clinical measurement. Lancet 1986;2:307-10.

17. Ge J, Erbel R, Gerber T, Gorge G, Koch L, Haude M, et al. Intravascular ultrasound imaging in angiographically normal coronary arteries: a prospective study in vivo. Br Heart $J$ 1994;71:572-8.

18. Hodgson JM, Reddy KG, Suneja R, Nair RN, Lesnefsky EJ, Sheehan HM. Intracoronary ultrasound imaging: correlation of plaque morphology with angiography, clinical syndrome and procedural results in patients undergoing coronary angioplasty. J Am CoIl Cardiol 1993;21:3544.

19. Losordo DW, Rosenfield K, Kaufman J, Pieczek A, Isner JM. Focal compensatory enlargement of human arteries in response to progressive atherosclerosis: in vivo documentation using intravascular ultrasound. Circulation 1994;89:2570-7. 
20. St. Goar FG, Pinto FJ, Alderman EL, Valantine HA, Schroeder JS, Gao SZ, et al. Intracoronary ultrasound in cardiac transplant recipients: in vivo evidence of 'angiographically silent' intimal thickening. Circulation 1992;85:979-87.

21. Mintz GS, Potkin BN, Keren G, Satler LF, Pichard AD, Kent KM, et al. Intravascular ultrasound evaluation of the effect of rotational atherectomy in obstructive atherosclerotic coronary artery disease. Circulation 1992;86:1383-93.

22. Nakamura S, Colombo A, Gaglione A, Almagor Y, Goldberg SL, Maiello $\mathrm{L}$, et al. Intracoronary ultrasound observations during stent implantation. Circulation 1994:89:2026-34.

23. Tenaglia AN, Buller CE, Kisslo KB, Stack RS, Davidson CJ. Mechanisms of balloon angioplasty and directional coronary atherectomy as assessed by intracoronary ultrasound. J Am Coll Cardiol 1992;20:68591.

24. Tobis JM, Mallery JA, Gessert J, Griffith J, Mahon D, Bessen M, et al. Intravascular ultrasound cross-sectional arterial imaging before and after balloon angioplasty in vitro. Circulation 1989;80:873-82.

25. Di Mario C, Gil R, Camenzind E, Ozaki Y, von Birgelen C, Umans V, et al. Quantitative assessment with intracoronary ultrasound of the mechanisms of restenosis after percutaneous transluminal coronary angioplasty and directional coronary atherectomy. Am J Cardiol 1995; 75:772-7.

26. Sonka M, Zhang X, Siebes M, DeJong S, McKay CR, Collins SM. Automated segmentation of coronary wall and plaque from intravascular ultrasound image sequences. In: Computers in cardiology 1994. Los Alamitos, Calif.: IEEE Computer Society Press, 1994:281-4.

27. Haase J, Ozaki Y, Di Mario C, Escaned J, De Feyter PJ, Roelandt JRTC, et al. Can intracoronary ultrasound correctly assess the luminal dimensions of coronary artery lesions? A comparison with quantitative angiography. Eur Heart J 1995;16:112-119.

28. Matar FA, Mintz GS, Douek P, Farb A, Virmani R, Saturnino PJ, et al. Coronary artery lumen volume measurement using three-dimensional intravascular ultrasound: validation of a new technique. Cathet Cardiovasc Diagn 1994;33:214-20.

29. Rosenfield K, Losordo DW, Ramaswamy K, Pastore JO, Langevin RE, Razvi S, et al. Three-dimensional reconstruction of human coronary and peripheral arteries from images recorded during two-dimensional intravascular ultrasound examination. Circulation 1991;84:193856

30. Cavaye DM, White RA, Lerman RD, Kopchok GE, Tabbara MR, Corm- ier F, et al. Usefulness of intravascular ultrasound imaging for detecting experimentally induced aortic dissection in dogs and for determining the effectiveness of endoluminal stenting. Am J Cardiol 1992; 69:705-7.

31. Coy KM, Park JC, Fishbein MC, Laas T, Diamond GA, Adler L, et al. In vitro validation of three-dimensional intravascular ultrasound for the evaluation of arterial injury after balloon angioplasty. J Am Coll Cardiol 1992;20:692-700.

32. Mintz GS, Pichard AD, Satler LF, Popma JJ, Kent KM, Leon MB. Three-dimensional intravascular ultrasonography: reconstruction of endovascular stents in vitro and in vivo. J Clin Ultrasound 1993;21:60915.

33. Schryver TE, Popma JJ, Kent KM, Leon MB, Eldredge S, Mintz GS Use of intracoronary ultrasound to identify the 'true' coronary lumen in chronic coronary dissection treated with intracoronary stenting. Am J Cardiol 1992;69:1107-8.

34. Chandrasekaran K, Sehgal CM, Hsu TL, Young NA, D'Adamo AJ, Robb RA, et al. Three-dimensional volumetric ultrasound imaging of arterial pathology from two-dimensional intravascular ultrasound: an in vitro study. Angiology 1994;45:253-64.

35. von Birgelen C, Slager CJ, Di Mario C, de Feyter PJ, Serruys PW. Volumetric intracoronary ultrasound: a new maximum confidence approach for the quantitative assessment of progression-regression of atherosclerosis? Atherosclerosis 1995;118(Suppl):S103-13.

36. Dhawale PJ, Wilson DL, Hodgson JM. Optimal data acquisition for volumetric intracoronary ultrasound. Cathet Cardiovasc Diagn 1994;32:288-99.

37. Bruining N, von Birgelen C, Di Mario C, Prati F, Li W, Den Hood W, et al. Dynamic three-dimensional reconstruction of ICUS images based on an ECG-gated pull-back device. In: Computers in cardiology 1995. Los Alamitos, Calif.: IEEE Computer Society Press, 1995;633-6.

38. Slager CJ, Laban M, von Birgelen C, Krams R, Oomen JAF, den Boer A, et al. ANGUS: a new approach to three-dimensional reconstruction of geometry and orientation of coronary lumen and plaque by combined use of coronary angiography and IVUS [Abstract]. J Am Coll Cardiol 1995;25(suppl):144A

39. Koch L, Kearney P, Erbel R, Roth T, Ge J, Brennecke R, et al. Three dimensional reconstruction of intracoronary ultrasound images: roadmapping with simultaneous digitised coronary angiograms. In: Computers in cardiology 1993. Los Alamitos, Calif.: IEEE Computer Society Press, 1993:89-91. 